\title{
Aesthetic Quality Classification of Photographs: A Literature Survey
}

\author{
Anu Joy \\ Department of Computer Science \\ College of engineering Poonjar
}

\author{
Sreekumar K \\ Department of Computer Science \\ College of engineering Poonjar
}

\begin{abstract}
Aesthetic quality classification plays an important role in our day to day life. Aesthetic generally refers to the beauty or the appreciation of beauty. Image aesthetic is a special session in the current area of computational visual aesthetic. Owing to the booming development of digital media the visual data available on the internet is growing exponentially. Some images are extremely beautiful and aesthetically pleasing. The aesthetic beauty of a picture is determined by many factors, some of these aesthetic quality factors include cuteness, prettiness, messiness, neatness, cuddleness, loveliness, organized, disorganized. It creates radical change in the sweet aesthetic sensations in both physiological and psychological. Many people these days have large photo collections. The aesthetic quality classification of photographs helps peoples to organize large photo collections. An aesthetic picture may tell an entire story of an event. Through a quality image we can see into the life of things. Therefore the assessment of image quality is a challenge. This paper gives a survey of aesthetic quality classification of photographs including the image quality factors, quality assessment and a brief description of existing approaches are presented.
\end{abstract}

\section{General Terms}

Algorithms, Experimentation

\section{Keywords}

Machine Learning, Photo Aesthetic Quality Assessment, Feature Extraction, Classification

\section{INTRODUCTION}

As a result of modern technology and extensive use of digital camera taking photograph is not at all a problem today. We take so many photographs of all events in our day today life. More than 350 million photographs are daily uploaded globally in social medias. Visual images have astounding influence in the field of journalism and communication. The quality of images differs very much. There are best quality images and worst quality images. Therefore the assessment of image quality is a challenge.

Many researchers have proposed aesthetic quality classification methods and aesthetic quality find applications in image cropping and recomposition. [7]The applications of aesthetic models have many practical values, in image retrieval systems similar images (from a content based perspective) could be reranked using aesthetic properties. They could help a user to select a best picture from his or her collections to make photo albums. And these models could be deployed directly in photo cameras to make real time suggestions or software that can help user to aesthetically design albums, slide shows and other photo related products. The aesthetic quality measures are advantageous because they are more closely correlated to our impression of images.
Image quality is a characteristic of an image that measures the perceived image degradation (typically, compared to an ideal or perfect image). Image quality is the merit and excellence of an image and the impression and influence created with reference to the subject as perceived by an expert or a computational expert.

Joshi et al [1] the American Heritage Dictionary of the English Language provides the following currently used definitions of aesthetics:

i. the branch of philosophy that deals with the nature and expression of beauty, as in the fine arts. In Kantian philosophy, the branch of metaphysics concerned with the laws of perception

ii. the study of the psychological responses to beauty and artistic experiences

iii. a conception of what is artistically valid or beautiful

iv. an artistically beautiful or pleasing appearance

\section{IMAGE QUALITY FACTORS}

i. Color Palette (CP) :[7]A good combination of colors within an image is related to visual attractiveness ,such color combinations are called color palette. Color accuracy is an important image quality factor but ambiguous. Majority of viewers prefer enhanced color saturation. The most accurate color need not be the most pleasing. It is very important to measure a camera's color response such as its color shifts, saturation, and the effectiveness of its white balance algorithms.

ii. Texture: measures look for visual patterns in images and how they are spatially defined.ie how an object feels or how it looks like it feels.

iii. Sharpness: determines the amount of detail an image can convey. System sharpness is affected by the lens and sensor (pixel count and anti-aliasing filter)

iv. Composition: it is the placement or arrangement of visual elements in an image as distinct from the subject of a work. layout and edge composition features are utilized in [7]

v. Content: equals to the humans understanding on the content of the image i.e, what the image depicts and what emotion it express.

vi. Noise is the random distraction or variation of image density. Usually appeared as grain in film and variations in digital images pixel level. There is noise reduction (NR) software that reduces the visibility of noise by smoothing the image, except the areas near contrast boundaries. But this 
smoothening technique may cause losing of image contrast Tone reproduction is the relationship between scene luminance and the reproduced image brightness.

vii. Contrast of images gives more clear picture quality. It is due to the tone reproduction curve in a $\log$-log- space. When the contrast is very high it causes loss of detail of the image.

viii. Distortion of image causes an anomaly in the way a straight line features are displayed with a curve. It affects the quality of the image while taking Architectural photos and in cases which involves measuring. It is most commonly visible in low cost cameras, cell phone cameras etc. Nowadays the Distortion anomaly can be corrected using sophisticated software's which will clear the image of all aberrations.

ix. Exposure accuracy: Some images taken from auto focus cameras the exposures will not be very good. This is due the fact that there is very little time for post- exposure tonal adjustments angle lenses.

x. Lateral Chromatic aberration (LCA): There are some cases where the color of the image gets a 'purple' tinge due to the aberration of the lens. This lens aberration forces the lens to focus on the object at different distances from the center of the object thereby causing a phenomenon called LCA. Cameras having asymmetrical lens with ultra wide true telephoto zooms are very susceptible to LCA

xi. Lens flare: The creations of 'ghost' images in some images are called lens flare. Lens flare occurs when the light gets reflected between the lens elements and the camera barrel. It can cause the image to appear to be fogging wherein the image loses its shadow detail and color.

xii. Color moiré: Some images display artificial color banding particularly in the case of fabrics and picket fences. This is called Color moiré and it is due lens sharpness, demosaicing software and anti-aliasing filter.

xiii. Artifacts: The quality tends to be very poor in cameras having sharp lenses. After the image is taken for processing in the software it may sometimes undergo compressing, loss of data during transmission, appearance of halos etc. These are called as artifacts.

\section{QUALITY ASSESSMENT}

In multimedia applications image quality is addressed by an integrated collection of tools such as image quality tools(full reference, no reference), image analysis or pattern recognition tools, $\mathrm{db}$ and textual image quality tools. There are direct and indirect methods are used to assess the quality of an image. In direct method

i. Psychophysical experiments with the participation of human experts(subjective)

ii. Computing suitable image qualities directly from the digital image(Objective)

According to [2],[12] Image quality can be indirectly assessed by i. Quantifying the performance of an image-based task done by the domain expert and/or by a computational system (objective).

ii. The performance of the imaging /rendering device on suitable set of target image is assessed by Using ad-hoc designed software tools one or more direct methods (objective/subjective).

[13] Full-reference (FR) metrics perform a direct comparison between the image under test and a reference or "original" in a properly defined image space. The requirement of the original is a restriction on the usability of such metrics. Just image fidelity can be assessed. We assume that image quality can be determined without a direct comparison between the original and processed image .No-reference (NR) metrics look only at the image under test and have no need of reference information.

Several factors affecting the aesthetic quality metric. Most of these are based on high level feature related to beauty such as colorfulness, line orientation, shape etc and rule of thumb such as composition, rule of third, skyline etc. The two famous aesthetic rules in the photography literature are Rule Of Thirds and Visual Weight Balance.

Rule of third is a high level photographic rule. In terms of composition it is the most well known theory. According to this theory the main element or the center of interest in a photograph is not the exact center of the photograph, but rather it should lie at one of the four intersections and at the one third and two third lines (both horizontal and vertical). The degree of adherence to this rule is measured by averaging the hue, saturation and intensities within the inner third region of the photograph. Pictures with simplistic composition and a well focused center of interest are more pleasing than pictures with many different objects.

\section{EXISTING APPROACHES}

Tsung et al[3] proposed a method for image quality assessment using multi method fusion. It integrate the scores obtained from multiple quality indices in to one score. Here it adopt a support vector regression approach, which is a machine learning tool used for fusion of multiple methods. In order to reduce the complexity of MMF ,a fused IQA method selection algorithm called Biggest Index Ranking Difference (BIRD) is developed. SFMS and BIRD algorithms are used to reduce the number of fused methods and lower the complexity of MMF. In most cases it need 3 methods for the fusion to achieve satisfactory performance,and the computational time is around 1minute per image .It was shown by experimental result that the approach performs better than well-known image quality indices.

Anish et al[4] proposed a completely blind IQA model called Natural Image Quality Evaluator(NIQE).This model is based on a collection of quality aware features and fitting them in to a Multivariate Gaussian (MVG)model. The quality aware features are based on a simple and successful space domain Natural Scene Static(NSS)model. These features are derived from a corpus of natural, undistorted images. The quality of the distorted image is expressed as a distance metric between the model statistics and those of the distorted image. It was shown by experimental results this model outperforms FR IQA models and competes with top performing NR IQA trained on human judgment of known distorted images. 
Hsiao et al [5] proposed a bottom up aesthetic modeling method for scenic photograph .This approach constructs an aesthetic library called bag-of -aesthetics-preserving (BoAP) feature image representation. It overcome both implicit and explicit aesthetic features with learning process. The BoAP features are extracted from different feature spaces to model color, texture, saliency and edge information jointly, and the extracted features constitute the feature set. Experimental results shows that this method $(92.06 \%$ in accuracy)performs better than state-of-the-art rule specific methods. Moreover among the selected features in the model contrast features are more effective than absolute features.

Luca et al [6] focus on generic image descriptor to assess aesthetic quality. Here it use generic content -based local image signature for aesthetic assessment. To encode the distribution of local statistics it use two families of generic image descriptors the Bag-of-Visual-Words and fisher vector. For both descriptors it encode gradient information using SIFT and color information. It also use GIST descriptor for scene categorization.

Kuo et al[7] proposed a method for designing a set of features that follows an instance -based approach. Method is implemented and visualized in online aesthetic assessment systems. Features focus on are based on color composition and spatial composition such as color palette, layout/edge composition, and global texture features. Classification Performance is evaluated using classification Accuracy (ACC).Area Under the ROC Curve is used to measure unbalanced dataset. Experimental results show that using simple technique is sufficient to reach great classification performance on different variability of photos.

Xin et al [8] focus on deep neural network approach to compute an automatic feature learning of the input image. The methodology used is RAPID system. Aesthetic quality rating and categorization is done using double column deep convolutional neural network approach. Here the central idea is to incorporate heterogeneous inputs generated from the image, which include a global view and a local view, and to unify the feature. The experimental results shows that this approach outperforms the existing methods on the AVA dataset.

Cong cong et al[9] focus on a machine learning scheme to calculate aesthetic visual quality of digital images of paintings. Feature extraction focus on color and composition. Color features are based on HSL space. Composition features are analyzed through the analysis on shapes and spatial relationship of different parts inside the image. Propose 40 features and form a feature set .Global and local features are considered. Global characteristics measured by classic models. Local characteristics measured by a specific metrics based on segments.

Christel et al [10] The proposed method provide a no reference perceptual harmony-guided quality map as well as a score of disharmony. Present a harmony quality assessment method to define what is visible or not in images and videos. Techniques based on human visual system models use signal masking to define visibility thresholds. Color rules are used to detect what part of images are disharmonious, and visual masking is applied to estimate to what extent an image area can be perceived disharmonious.

Xiaoou et al [11], [14] Propose a content-based photo quality assessment frame work. Here in order to assess photos in different categories both regional and global features are selected and combined. Three methods are proposed to extract subject areas, which are clarity based region detection, layout based region detection and human based detection. The features are emphasizing on dark channel feature, hue composition feature and human based features. The features are combined with an SVM classifier. It was shown experimental result that their effectiveness highly depends on the visual content.

Table 1. Comparison of Different Aesthetic Image Quality Methods

\begin{tabular}{|c|c|c|c|c|c|c|}
\hline Method & Author & $\begin{array}{c}\text { Year of } \\
\text { Publication }\end{array}$ & $\begin{array}{c}\text { Learning } \\
\text { Method }\end{array}$ & Accuracy & Advantages/Disadvantages & Review \\
\hline MMF [3] & $\begin{array}{l}\text { Tsung-Jung Liu, } \\
\text { Weisi Lin, C.- } \\
\text { C.Jay Kuo }\end{array}$ & 2013 & SVR & $\begin{array}{l}\text { On PLCC } \\
\text { by using } 3 \\
\text { methods } \\
0.9307(C \\
\text { F-MMF) } \\
\& \\
0.9517(\mathrm{C} \\
\text { D-MMF) }\end{array}$ & $\begin{array}{l}\text { i) For satisfactory } \\
\text { performance only } 3 \text { methods } \\
\text { are needed } \\
\text { ii)Flexibility to include other } \\
\text { methods } \\
\text { iii)Excellent performance }\end{array}$ & $\begin{array}{l}\text { Here a regression } \\
\text { technique is used } \\
\text { for estimating the } \\
\text { quality score, } \\
\text { which is seemed to } \\
\text { be good. } \\
\text { Automatic } \\
\text { detection of } \\
\text { context need to be } \\
\text { improved }\end{array}$ \\
\hline $\begin{array}{l}\text { NIQE(Natural } \\
\text { Image Quality } \\
\text { Evaluator) [4] }\end{array}$ & $\begin{array}{l}\text { Anish } \\
\text { Mittal,Rajiv } \\
\text { Soundararajan } \\
\text { and Alan C } \\
\text { Bovik }\end{array}$ & 2012 & $\begin{array}{l}\text { Not } \\
\text { applicable }\end{array}$ & $91.47 \%$ & $\begin{array}{l}\text { i)NIQE performs better than } \\
\text { the FR PSNR and SSIM } \\
\text { ii)remarkable demonstration } \\
\text { of the relationship between } \\
\text { quantified image naturalness } \\
\text { and perceptual image quality }\end{array}$ & $\begin{array}{l}\text { There may be } \\
\text { some overlap } \\
\text { occurs between } \\
\text { train and test } \\
\text { content, so careful } \\
\text { attention is needed }\end{array}$ \\
\hline
\end{tabular}




\begin{tabular}{|c|c|c|c|c|c|c|}
\hline $\begin{array}{l}\text { Bottom -up } \\
\text { aesthetic } \\
\text { modeling } \\
\text { method [5] }\end{array}$ & $\begin{array}{l}\text { Hsiao-Hang } \\
\text {,Su,Tse-wei chen } \\
\text {, chieh-chi } \\
\text { kao,Winston H } \\
\text { Hsu, shao-Yi } \\
\text { Chien }\end{array}$ & 2011 & $\begin{array}{l}\text { Bag-of - } \\
\text { Aesthetics } \\
\text { Preserving }\end{array}$ & $92.06 \%$ & $\begin{array}{l}\text { i) Rule specific features are } \\
\text { covered } \\
\text { ii) In the decomposition } \\
\text { method image can be viewed } \\
\text { in multiple resolutions } \\
\text { iii) Images can be described } \\
\text { from different aesthetic } \\
\text { aspects including color, } \\
\text { texture, saliency and edge } \\
\text { iv) Contrast information } \\
\text { taken in to consideration } \\
\text { v) Computation process is } \\
\text { highly efficient }\end{array}$ & $\begin{array}{l}\text { Instead of giving } \\
\text { aesthetic score as } \\
\text { binary ,multiple } \\
\text { scores can be } \\
\text { given }\end{array}$ \\
\hline $\begin{array}{l}\text { Generic image } \\
\text { descriptor [6] }\end{array}$ & $\begin{array}{l}\text { Luca } \\
\text { Marchesotti, } \\
\text { Florent } \\
\text { Perronnin, Diane } \\
\text { Larlus, Gabriela } \\
\text { Csurka }\end{array}$ & 2011 & Linear SVM & $\begin{array}{l}\text { ACC- } \\
86 \% \\
\text { AUC- } \\
93 \%\end{array}$ & $\begin{array}{l}\text { i) Acceptable computation } \\
\text { times for nearly real-time } \\
\text { applications. } \\
\text { ii) Classification rates are } \\
\text { significantly better } \\
\text { iii) More potential to be } \\
\text { carried out in instant manner } \\
\text { on mobile or embedded } \\
\text { devices for future } \\
\text { applications }\end{array}$ & $\begin{array}{l}\text { Blur information } \\
\text { can also be added } \\
\text { to the color } \\
\text { descriptors } \\
\text { The addition of } \\
\text { local geometry to } \\
\text { the color } \\
\text { descriptors can } \\
\text { extract information } \\
\text { regarding edges, } \\
\text { contrast and } \\
\text { sharpness }\end{array}$ \\
\hline RAPID [8] & $\begin{array}{l}\text { Xin Lu,Zhe Lin, } \\
\text { Jian chaoYang, } \\
\text { James Z }\end{array}$ & 2014 & $\begin{array}{l}\text { Double - } \\
\text { column } \\
\text { Deep } \\
\text { Convolution } \\
\text { al Neural } \\
\text { Network }\end{array}$ & $71.20 \%$ & $\begin{array}{l}\text { i) For judging its aesthetic } \\
\text { quality both global and local } \\
\text { views of an image taken in } \\
\text { to account. }\end{array}$ & $\begin{array}{l}\text { Neural network } \\
\text { approach is very } \\
\text { good. } \\
\text { It take more time } \\
\text { for computation, } \\
\text { so acceptable } \\
\text { computational } \\
\text { times are needed }\end{array}$ \\
\hline $\begin{array}{l}\text { Data - driven } \\
\text { machine } \\
\text { learning } \\
\text { problem }\end{array}$ & $\begin{array}{l}\text { Congcong } \\
\text { Li,Tsuhan Chen }\end{array}$ & 2013 & $\begin{array}{l}\text { Naïve Bayes } \\
\text { \& Adaptive } \\
\text { Boosting } \\
\text { Classifier }\end{array}$ & $87 \%$ & $\begin{array}{l}\text { i) Both classifiers are robust } \\
\text { to produce good accuracy } \\
\text { using the } 40 \text { extracted visual } \\
\text { features in discriminating } \\
\text { high-rated and low-rated } \\
\text { paintings }\end{array}$ & $\begin{array}{l}\text { A regression } \\
\text { approach can be } \\
\text { used to estimate } \\
\text { the quality score }\end{array}$ \\
\hline
\end{tabular}

\section{CONCLUSION}

The invention of digital cameras capturing images has become extremely easy. Due to the exponential increase of consumer photos, evaluating the quality of photos has become a more complicated topic. Many image hosting and sharing website have proved the need of introducing some form of aesthetic or appeal measures. Aesthetic appeal can be useful in finding out exciting and appealing photographs from large collections while looking for unappealing one. This paper gives a survey of aesthetic quality classification of photograph, which helps general readers have an overview of the need of image aesthetic quality, the image quality factors. The quality assessment and existing approaches are also discussed. From this survey we can propose a method for aesthetic quality classification of photographs using double column deep convolutional neural network approach as learning technique [8] with feature extraction technique used in [9], and also instead of finding the aesthetic quality score as binary(good or bad),we can find multiple aesthetic quality scores (excellent,good,average,bad) as a future work.

\section{REFERENCES}

[1] Dhiraj Joshi, Ritendra Datta,Elena Fedorovskaya,QuangTuan Luong,James Z. Wang,Jia Li, and Jiebo Luo 2011 Aesthetic and Emotions in Image.

[2] G. Ciocca, F. Marini, R. Schettini Image quality in multimedia applications

[3] Tsung-Jung Liu, Weisi Lin, C.-C.Jay Kuo image quality assessment using multi method fusion 2013 IEEE Transactions on Image processing,VOL.22,No.5,May 2013

[4] Anish Mittal,Rajiv Soundararajan and Alan C Bovik 2012 Making a 'completely blind' image quality analyzer IEEE

[5] Hsiao-Hang ,Su,Tse-wei chen , chieh-chi kao,Winston $\mathrm{H}$ Hsu ,shao-Yi Chien 2011 Scenic photo quality assessment with bag of aesthetic -preserving features, $\mathrm{ACM}$

[6] Luca Marchesotti,Florent Perronnin,Diane Larlus,Gabriela Csurka 2011 Assessing the aesthetic 
quality of photographs using generic image descriptors IEEE international conference on computer vision.

[7] Kuo-Yen Lo,Keng-Hao Liu and Chu-Song Chen assessment of Photo Aesthetic with Efficiency

[8] Xin Lu,Zhe Lin, Jian chaoYang,James Z wang 2014. RAPID:RAting PIctorial aesthetic using Deep learning.In ACM

[9] Congcong Li,Tsuhan Chen Aesthetic Visual Quality Assessment of Paintings IJSTSP

[10] Christel Chamaret and Fabrice Urban 2013 NoReference Harmony-guided Quality Assessment IEEE Conference on Computer Vision And Pattern Recognition Workshops
[11] Xiaoou Tang,Wei Luo and Xiaogang Wang 2013 content-Based photo quality assessment IEEE.

[12] Dr.Mohammed Nasir uddin, Dr.Jebunnahar and Md.Abul Bashar 2012. A Comprehensive Study of Digital Image Processing for Finding Image Quality Dependencies.IJSRP

[13] Fabrizio Marini Content Based No-Reference Image Quality metrics

[14] Xiaoou Tang,Wei Luo and Xiaogang Wang 2011 content-Based photo quality assessment 